Psychotherapy and

Psychosomatics

\section{Announcement}

Psychother Psychosom 2021;90:287

DOI: 10.1159/000517379
Published online: June 3, 2021

\title{
Introductory Course on Well-Being Therapy
}

November 5-7, 2021

A specific psychotherapeutic strategy for modulating psychological well-being and pursuing euthymia, Well-Being Therapy (WBT), has been developed and validated in a number of randomized controlled trials. WBT has been manualized [Fava GA: Well-Being Therapy. Treatment Manual and Clinical Applications. Basel, Karger, 2016]. Translations of the manual have been published in Italian, Portuguese, Chinese, Japanese, German, French, Hungarian, and Turkish. A certification process for WBT has been promoted by the Academy of Well-Being Therapy (AWBT), whose president is Giovanni A. Fava. The certification process includes attendance at an introductory course, individual supervision of two cases treated with WBT, and case presentation at an advanced workshop. The next introductory course on Well-Being Therapy will be held online on November 5-7, 2021.

For further information please visit www.well-being-therapy.com. 